\title{
Bedside or Surgical Fasciotomy: Which would You Choose for Treatment of Compartment Syndrome?
}

\author{
Mohammad Rimawi ${ }^{1}$, Jose M Gonzalez ${ }^{2}$
}

\begin{abstract}
A compartment is an area of muscle groups and neurovascular structures enclosed by fascia. The leg consists of four compartments: anterior, lateral, superficial posterior, and deep posterior. The foot, although occupying less surface area than the leg, contains nine compartments. Common causes include but are not limited to trauma, hemorrhage, tight casting, or constrictive bandaging. When the physician has diagnosed an acute compartment syndrome, surgical decompressive fasciotomy should be performed urgently. Although a compartmental release in the operating room is the standard of care, in select cases, bedside fasciotomy can be performed to reduce the incidence of morbidity in a patient. In this brief review, we discuss the use of a bedside fasciotomy under local anesthetics in select cases to avoid delay in compartmental release. Keywords: Bedside, Bedside fasciotomy, Compartment, Compartment syndrome, Podiatric emergency. Journal of Foot and Ankle Surgery (Asia-Pacific) (2019): 10.5005/jp-journals-10040-1100
\end{abstract}

A compartment is an area of muscle groups and neurovascular structures enclosed by fascia. The leg consists of four compartments: anterior, lateral, superficial posterior, and deep posterior. The foot, although occupying less surface area than the leg, contains nine compartments. The compartments as explained by Manoli and Weber ${ }^{1}$ are the following: medial, lateral, four interossei, calcaneal, and superficial and deep central compartments. Compartment syndrome is defined as a raised pressure within an enclosed area with the potential to cause irreversible damage to its contents. This ability to permanently damage these structures is what makes compartment syndrome a podiatric surgical emergency. Therefore, it is important for one to be able to efficiently identify, diagnose, and treat a compartment syndrome. In this updated chapter, the authors will discuss the use of a bedside fasciotomy under local anesthetics in select cases to avoid delay in compartmental release.

Before treating compartment syndrome, one must be able to properly identify the condition. Common causes include but are not limited to trauma, hemorrhage, tight casting, or constrictive bandaging. If a patient presents with a cast or dressing, it is advised to remove the causative agent. Garfin and Mubarak cited an average reduction of $65 \%$ in compartmental pressures after removal of a cast, and then an additional decrease of $10-20 \%$ after the dressing was removed. ${ }^{2}$ The presence of an open fracture does not exclude the diagnosis of compartment syndrome. Although the affected area is open, the adjacent compartments are not necessarily spared. The historical symptoms of a patient with compartment syndrome are the following: pain, pallor, paresthesia, paralysis, and pulselessness. However, these symptoms should not be considered a reliable finding. A patient with an early presentation will have pulses and an adequate capillary fill time. The vascular deficiency tends to be a late finding of compartment syndrome. Immediate symptoms that should warrant a compartment syndrome are allodynia and palpable tenseness in the involved compartment.

Compartment syndrome can only be accurately diagnosed with the use of a Wick's catheter. There are several different theories cited in the literature that can aid with the interpretation of the results. The first, being the absolute pressure theory presented by Mubarak ${ }^{3}$ and Matsen, ${ }^{4}$ who suggested a fasciotomy be performed when intracompartmental pressures reach or exceed $30 \mathrm{~mm} \mathrm{Hg}$

\begin{abstract}
1,2Department of Podiatry, Dekalb Medical, Decatur, Illinois, USA
Corresponding Author: Jose M Gonzalez, Department of Podiatry, Dekalb Medical, Decatur, Illinois, USA, e-mail: jmgonzal12@gmail.com How to cite this article: Rimawi M, Gonzalez JM, et al. Bedside or Surgical Fasciotomy: Which would You Choose for Treatment of Compartment Syndrome? J Foot Ankle Surg (Asia-Pacific), 2019;6(1):23-24.
\end{abstract}

Source of support: Nil

Conflict of interest: None

(Mubarak) and $45 \mathrm{~mm} \mathrm{Hg}$ (Matsen). Then, there is the perfusion theory of Whitesides, ${ }^{5}$ who revealed the relationship between tissue perfusion and diastolic blood pressure (DBP). He recommended surgical decompression when the tissue pressure is within $20 \mathrm{~mm}$ $\mathrm{Hg}$ of the DBP. McQueen ${ }^{6}$ suggested a differential $<30 \mathrm{~mm} \mathrm{Hg}$ of the diastolic pressure and the intramuscular pressure as a threshold for release as being more reliable.

\section{Technique}

When the physician has diagnosed an acute compartment syndrome, surgical decompressive fasciotomy should be performed urgently. There are various techniques for fasciotomy involving the leg that have been described. They include single-incision fasciotomy without fibulectomy, single incision with fibulectomy, and the more common two incision fasciotomies.

The single-incision technique entails a longitudinal incision made over the fibula extending $5 \mathrm{~cm}$ distal to the fibular head and $5 \mathrm{~cm}$ proximal to the lateral malleolus. The anterior, lateral, and superficial posterior compartments are released first. This is then followed by the release of the deep posterior compartment at the posterolateral fibular insertion site of the lateral intermuscular septum. This approach is less favorable due to the risk involved of damaging the peroneal nerves and vessels when entering the deep posterior compartment. Fibulectomy can be performed through this operative technique; however, this technique is rarely used due to the two incision fasciotomies causing less morbidity. ${ }^{7}$

In the two-incision technique, the anterolateral incision is made to approach the anterior and lateral compartments. This 
incision is placed between the tibial crest and the head of the fibula. The incision is very much the same one used for the singleincision technique. One should be able to release the fascia of the anterior and lateral compartments through this incision. Care must be taken by the performing surgeon to avoid the superficial peroneal nerve, which can be encountered $10-12 \mathrm{~cm}$ proximal to the lateral malleolus. The second incision is the posteromedial incision. The incision is made $2 \mathrm{~cm}$ posterior to the medial border of the tibia. With this incision, the superficial and deep posterior compartments can be assessed for any soft tissue compromise. Surgeons should avoid sacrificing the saphenous nerve and the vein while performing this procedure.

The most common approach to compartment syndrome of the foot remains a combined dorsal and medial plantar incisions. ${ }^{8}$ The medial incision starts at the medial side of the calcaneus just anterior to the abductor hallucis origin, extending distally, parallel to the plantar surface for $6 \mathrm{~cm}$. Care must be taken to avoid damage to the medial and lateral plantar nerve and vessels through this incision. Next, two separate dorsal incisions are made. These incisions are placed medial to the second metatarsal and lateral to the fourth, running longitudinal to the respective metatarsals. ${ }^{9}$

Closure of the aforementioned wounds by secondary intention is generally indicated after 5-7 day's post-fasciotomy. ${ }^{10}$ Skin grafting may be indicated in the event that skin closure cannot be performed. Due to medial incision often times being difficult to close, open reduction internal fixation of the calcaneus should be attempted 10-14 days post-fasciotomy. This prevents any risk of leaving exposed hardware that in turn can lead to infection.

\section{Alternative Treatment}

Although a compartmental release in the operating room is the standard of care, in select cases, bedside fasciotomy can be performed to reduce the incidence of morbidity in a patient. ${ }^{11}$ This alternative method can be used in patients with a delayed presentation of compartment syndrome, or where a delay in surgery is anticipated. Antibiotic prophylaxis is appropriately administered and continued for 24 hours post-fasciotomy. The affected extremity can then be prepped and draped in the normal sterile fashion. Conscious sedation consisting of midazolam and fentanyl along with $1 \%$ lidocaine with epinephrine along incisions is used to perform release of compartments. Fasciotomy can then be performed using the above-mentioned techniques. Any subsequent procedures besides the fasciotomy can then be done in the operating room after the patient has been stabilized. Saline-soaked wet to dry dressings on the site of fasciotomy can be applied postoperatively. Any further wound irrigation and debridements are done as needed. Once the edema resolves, wounds are closed through delayed primary closure, negative pressure wound therapy assisted closure, or split-thickness skin grafting. ${ }^{12,13}$ Dr Ebraheim cited this alternative method in 2012 and reported a total of 34 cases in which it was used. He described immediate improvement in pain, with adequate wound healing and no cases of infection. Although the conventional release of compartments in the operating room continues to be the standard of care, a bedside fasciotomy under local anesthesia is a viable, safe, and effective option for treating compartment syndrome in select cases.

\section{References}

1. Manoli A, Weber TG. Fasciotomy of the foot: an anatomical study with special reference to release of the calcaneal compartment. Foot Ankle 1990 Apr;10(5):267-275. DOI: 10.1177/107110079001000505.

2. Garfin SR, Mubarak SJ, et al. Quantification of intracompartmental pressure and volume under plaster casts. J Bone Joint Surg Am 1981 Mar;63(3):449-453. DOI: 10.2106/00004623-198163030-00020.

3. Mubarak SJ, Owen CA, et al. Acute compartment syndromes: diagnosis and treatment with the aid of the Wick catheter. J Bone Joint Surg Am 1978 Dec;60(8):1091-1095. DOI: 10.2106/00004623197860080-00012.

4. Matsen FA, Winquist RA, et al. Diagnosis and management of compartment syndromes. J Bone Joint Surg Am 1980 Mar;62(2): 286-291. DOI: 10.2106/00004623-198062020-00016.

5. Matava MJ, Whitesides TE Jr. Determination of the compartment pressure threshold of muscle ischemia in a canine model. J Trauma 1994 Jul;37(1):50-58. DOI: 10.1097/00005373-199407000-00010.

6. McQueen MM, Court-Brown CM. Compartment monitoring in tibial fractures. The pressure threshold for decompression. J Bone Joint Surg Br 1996 Jan;78(1):99-104. DOI: 10.1302/0301-620X.78B1.0780099.

7. Maheshwari R, Taitsman LA, et al. Single-incision fasciotomy for compartmental syndrome of the leg in patients with diaphyseal tibial fractures. J Ortho Trauma 2008;22(10):723-730. DOI: 10.1097/ BOT.0b013e31818e43f9.

8. Myerson MS. Diagnosis and treatment of compartment syndrome of the foot. J of Ortho 1990;13(7):711-717.

9. Myerson MS. Experimental decompression of the fascial compartments of the foot- the basis for fasciotomy in acute compartment syndromes. J Foot Ankle 1988;8(6):308-314. DOI: 10.1177/ 107110078800800606.

10. Myerson MS, Manoli A. Compartment syndromes of the foot after calcaneal fractures. Clin Orthop 1993;290:142-150. DOI: 10.1097/00003086-199305000-00018.

11. Ebraheim NA, Abdelgawad AA. Bedside fasciotomy under local anesthesia for acute compartment syndrome: a feasible and reliable procedure in selected cases. J Ortho Trauma 2012;13(3):153-157. DOI: 10.1007/s10195-012-0196-9.

12. Morykwas MJ, Argenta LC, et al. Vacuum-assisted closure: a new method for wound control and treatment: animal studies and basic foundation. Ann Plast Surg 1997;38(6):553-562. DOI: 10.1097/00000637-199706000-00001.

13. Zannis J, Angobaldo J, et al. Comparison of fasciotomy wound closures using traditional dressing changes and the vacuum-assisted closure device. Ann Plast Surg 2009;62(4):407-409. DOI: 10.1097/ SAP.0b013e3181881b29. 